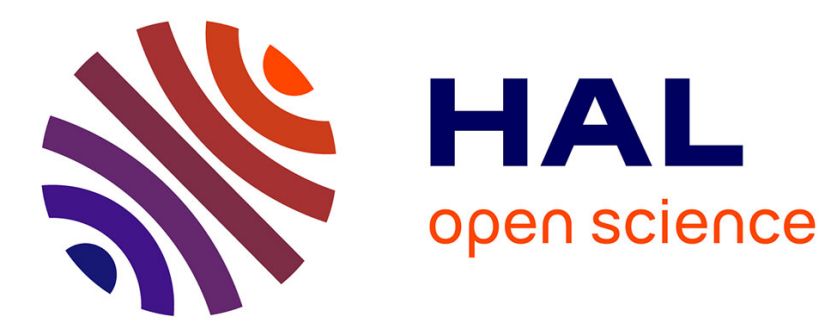

\title{
MIMO UWB Systems based on Linear Precoded OFDM for Home Gigabit Applications
}

Antoine Stephan, Jean-François Hélard, Bernard Uguen

\section{To cite this version:}

Antoine Stephan, Jean-François Hélard, Bernard Uguen. MIMO UWB Systems based on Linear Precoded OFDM for Home Gigabit Applications. IEEE Global Communications Conference (GLOBECOM'08), Dec 2008, New Orleans, United States. hal-00325635

\section{HAL Id: hal-00325635 \\ https://hal.science/hal-00325635}

Submitted on 29 Sep 2008

HAL is a multi-disciplinary open access archive for the deposit and dissemination of scientific research documents, whether they are published or not. The documents may come from teaching and research institutions in France or abroad, or from public or private research centers.
L'archive ouverte pluridisciplinaire HAL, est destinée au dépôt et à la diffusion de documents scientifiques de niveau recherche, publiés ou non, émanant des établissements d'enseignement et de recherche français ou étrangers, des laboratoires publics ou privés. 


\title{
MIMO UWB Systems based on Linear Precoded OFDM for Home Gigabit Applications
}

\author{
Antoine Stephan, Member, IEEE, Jean-François Hélard, Senior Member, IEEE, and Bernard Uguen \\ Institute of Electronics and Telecommunications of Rennes (IETR) \\ INSA, 20 Avenue des Buttes de Coesmes, 35043 Rennes, France \\ antoine.stephan@insa-rennes.fr, jean-francois.helard@insa-rennes.fr
}

\begin{abstract}
In this paper, we investigate the use of multipleinput multiple-output (MIMO) techniques with linear precoded orthogonal frequency division multiplexing (LP-OFDM) waveform for high data rate ultra-wideband (UWB) systems. This scheme is an evolution of the multiband OFDM (MB-OFDM) solution supported by the WiMedia Alliance. The aim of this paper is to obtain a very high data rate of around one gigabit for home access networks (HAN) and to improve the system range for lower data rates, while not significantly increasing the system complexity compared to the WiMedia solution. Firstly, a singleinput single-output (SISO) LP-OFDM system study is led to highlight the benefits of adding a precoding function to an OFDM signal in the UWB context. In an analytical study, different system choices and parameterization strategies are proposed in order to minimize the mean bit-error-rate (BER) and consequently improve the system range. Secondly, a MIMO scheme is added and global system simulations are performed on a proposed new geometric statistic MIMO channel model. We show that the proposed system can considerably improve the system range at low data rates, and can reach very high data rates up to $1 \mathrm{Gbit} / \mathrm{s}$ with comparable BER performances to WiMedia.
\end{abstract}

Index Terms - Linear precoded OFDM, Mean BER, MIMO, UWB, WiMedia.

\section{INTRODUCTION}

$\mathrm{U}$ LTRA-WIDEBAND (UWB) is an emerging technology that has attracted considerable interest in the research and standardization communities for wireless communications since the Federal Communications Commission (FCC) Report and Order in 2002 [1], when a 3.1-10.6 GHz spectrum was made available for unlicensed use of UWB devices. One of the main advantages of UWB is its ability to provide high data rate at low cost and relatively low power consumption. However, very stringent regulations were imposed on UWB since the 7.5 $\mathrm{GHz}$ allocated spectrum overlays other existing allo-

The research leading to these results has received funding from the European Community's Seventh Framework Programme FP7/2007-2013 under grant agreement $\mathrm{n}^{\circ} 213311$ also referred as OMEGA, and from France Telecom R\&D RESA/BWA within the contract 46136582. cated frequency bands. In order to reduce interference with existing services, the FCC imposed a power spectral density (PSD) limit of $-41.3 \mathrm{dBm} / \mathrm{MHz}$. One of the main multipleaccess techniques considered for high data rate UWB wireless personal area networks (WPAN) standardization is a multiband orthogonal frequency division multiplexing (MB-OFDM) supported by the MultiBand OFDM Alliance (MBOA) and the WiMedia forum [2], [3], which merged in March 2005 and are today known as the WiMedia Alliance.

The purpose of this paper is to improve the WiMedia system in terms of range and throughput by proposing a new UWB scheme based on the combination of linear precoding (LP) principles with the OFDM waveform of the WiMedia solution. The LP process consists in applying precoding matrices or equivalently spreading sequences to various blocks of subcarriers of the multicarrier spectrum [4]. In the resulting LPOFDM scheme, also known as spread spectrum multicarrier multiple-access (SS-MC-MA) in the wireless context [5], the OFDM parameters of the WiMedia solution are maintained in order not to increase the system complexity significantly. The LP-OFDM UWB system is first analyzed through a new analytical study that focuses on the optimization of the precoding function. In a second step, we move to a global LP-OFDM system study, taking into account the different functions of the transmission chain, and adding a multiple-input multipleoutput (MIMO) component to exploit the spatial diversity. In addition, a new realistic channel model based on a geometric statistic approach is proposed for MIMO-UWB applications. This system study, complementarily to the analytical one, points out the advantages of appropriately combining the MIMO and LP components with the WiMedia solution.

The rest of the paper is organized as follows. Section II briefly introduces the WiMedia solution and describes the proposed LP-OFDM system after discussing, from a general point of view, the interests of adding a LP component to the OFDM scheme. Section III details the analytical study that finds the optimal configuration of the precoding function that improves the system performance and minimizes the system mean BER. In Section IV, the added MIMO component and the proposed 
new MIMO-UWB channel model are described. Global system simulation results showing the interest of the proposed MIMO LP-OFDM system for UWB applications are given and interpreted in Section V. Finally, Section VI concludes the paper.

\section{SYSTEM DESCRIPTION}

\section{A. WiMedia solution}

The WiMedia solution combines OFDM with a multibanding technique that divides the UWB spectrum into 14 subbands of $528 \mathrm{MHz}$ each. An OFDM signal can be transmitted on each sub-band using a 128-point inverse fast Fourier transform (IFFT). Out of the 128 subcarriers used, only 100 are assigned to transmit data. The multiuser access is performed with time-frequency codes (TFC) which provide frequency hopping from a sub-band to another at the end of each OFDM symbol. The TFC allows every user to benefit from frequency diversity over a bandwidth equal to three sub-bands.

Different data rates from 53.3 to $480 \mathrm{Mbit} / \mathrm{s}$, listed in Table I, are obtained through the use of FEC, frequency-domain spreading (FDS) and time-domain spreading (TDS). The constellation applied to the different subcarriers is either a quadrature phase-shift keying (QPSK) for low data rates or a dual carrier modulation (DCM) for high data rates.

In brief, the WiMedia solution offers potential advantages for high data rate UWB applications, such as the signal robustness against channel selectivity and the efficient exploitation of the energy of every signal received within the prefix margin. However, it can be improved by combining precoding schemes with OFDM, as already seen in other contexts, such as cellular communications.

\section{B. Proposed LP-OFDM system}

In this paper, we propose to add a LP component to the WiMedia solution. The resulting LP-OFDM scheme is applied to UWB while respecting the OFDM parameters of the WiMedia solution. Hence, the system evolution reduces in practice to a simple addition of a precoding block in the transmission chain, which does not increase the system complexity significantly. Taking into account the frequency selectivity and the slow-time variations of the UWB channel in an indoor environment, the spreading sequences are applied in the frequency domain. This spreading component improves the signal robustness against frequency selectivity and narrowband interference, since the signal bandwidth can become much larger than the coherence and interference bandwidths. Moreover, it increases the resource allocation flexibility as the spreading code dimension offers an additional degree of freedom [6]. We will assume that orthogonal spreading sequences are used in the proposed system.

The LP-OFDM scheme is applied to UWB as follows. At a given time, each user is allocated one of the first three WiMedia sub-bands of $528 \mathrm{MHz}$ bandwidth each, in order not to increase the system complexity compared to the WiMedia solution. Each sub-band is then divided into several blocks, each
TABLE I

WIMEDIA SYSTEM DATA RATES

\begin{tabular}{ccccc}
\hline \hline $\begin{array}{c}\text { Data } \\
\text { rate } \\
(\mathrm{Mbit} / \mathrm{s})\end{array}$ & Modulation & $\begin{array}{c}\text { Convolutional } \\
\text { coding rate }(r)\end{array}$ & $\begin{array}{c}\text { Frequency- } \\
\text { domain } \\
\text { spreading }\end{array}$ & $\begin{array}{c}\text { Time- } \\
\text { domain } \\
\text { spreading }\end{array}$ \\
\hline 53.3 & QPSK & $1 / 3$ & Yes & Yes \\
80 & QPSK & $1 / 2$ & Yes & Yes \\
106.7 & QPSK & $1 / 3$ & No & Yes \\
160 & QPSK & $1 / 2$ & No & Yes \\
200 & QPSK & $5 / 8$ & No & Yes \\
320 & DCM & $1 / 2$ & No & No \\
400 & DCM & $5 / 8$ & No & No \\
480 & DCM & $3 / 4$ & No & No \\
\hline \hline
\end{tabular}

of them including a number of subcarriers equal to the spreading code length $L$. In addition, $C$ symbols are simultaneously transmitted by the same user on a specific subset of subcarriers and undergo the same distortions, with $C$ the number of used spreading sequences. It is well known that the spreading operation introduces some interference between the spreading sequences when orthogonality is not maintained. This selfinterference (SI) can actually be easily compensated for by a simple detection with only one complex coefficient per subcarrier.

\section{ANALYTICAL STUDY}

As mentioned in the introductive part, this section is dedicated to an analytical study around the LP-OFDM waveform. More precisely, the intrinsic characteristics and capabilities of the precoding function are highlighted. The precoding function can be viewed as a particular spread spectrum operation that brings some additional parameters which are the number $C$ and length $L$ of the spreading sequences used in the system, and the number $K$ of blocks. These parameters constitute new degrees of freedom in terms of system configuration and can be optimally selected. The objective of this study is to minimize the mean BER of a LP-OFDM system under a PSD constraint and a fixed target throughput using a QPSK constellation. In a first step, we optimize a single-block system, and then we move to a global multi-block system.

\section{A. Single-block system}

The throughput of a single-block LP-OFDM system in bit per symbol using a zero-forcing $(\mathrm{ZF})$ detection can be derived from Shannon theorem and leads to [7]

$$
R=\sum_{c=1}^{C} b_{c}=\sum_{c=1}^{C} \log _{2}\left(1+\frac{1}{\Gamma_{c}} \frac{L^{2}}{\sum_{i=1}^{L}\left(1 /\left|h_{i}\right|^{2}\right)} \frac{E_{c}}{N_{0}}\right),
$$

where $b_{c}$ is the number of bits allocated to code $c, \Gamma_{c}$ the signal-to-noise ratio (SNR) gap of the QAM constellation, $h_{i}$ the frequency-domain response for subcarrier $i, E_{c}$ the power density of code $c$, and $N_{0}$ the noise density.

Considering a fixed maximum target throughput with $C=L$, minimizing the mean BER is equivalent to 


$$
\min \frac{\sum_{c=1}^{L}\left(b_{c} P_{c}\right)}{\sum_{c=1}^{L} b_{c}} \text { subject to } \sum_{c=1}^{L} E_{c}=E,
$$

where $E$ is the PSD limit per block, and $P_{c}$ the BER for code $c$, which can be derived from [8] and expressed for a LP-OFDM system with a QPSK constellation as

$$
P_{c} \approx \frac{2}{b_{c}}\left(1-\frac{1}{b_{c}}\right) \operatorname{erfc}\left(\sqrt{\frac{3 S N R}{2\left(2^{b_{c}}-1\right)}}\right)=\frac{1}{2} \operatorname{erfc}\left(\sqrt{\frac{L^{2} E_{c} / 2 N_{0}}{\sum_{i=1}^{L}\left(1 /\left|h_{i}\right|^{2}\right)}}\right) .
$$

Applying Lagrange multipliers to (2) and (3) and using Lambert $W$ function, we obtain

$$
E_{c}=\frac{E}{L} \text { and } \Gamma_{c}=\frac{L E}{3 N_{0} \sum_{i=1}^{L}\left(1 /\left|h_{i}\right|^{2}\right)}, \quad \forall c .
$$

Consequently, the mean BER of a single-block is given by

$$
B E R_{\text {1-block }}=\frac{1}{2} \operatorname{erfc}\left(\sqrt{\frac{L E}{2 N_{0} \sum_{i=1}^{L}\left(1 /\left|h_{i}\right|^{2}\right)}}\right) .
$$

\section{B. Multi-block system}

Now, we study the case of a LP-OFDM system with $K$ blocks, all having the same code length $L$. Minimizing the total system mean BER is equivalent to

$$
\min _{K} \frac{1}{K} \sum_{k=1}^{K} \frac{1}{2} \operatorname{erfc}\left(\sqrt{\frac{L E}{2 N_{0} \sum_{i=1}^{L}\left(1 /\left|h_{k, i}\right|^{2}\right)}}\right) \text { with } K L=N \text {, (6) }
$$

where $h_{k, i}$ is the frequency-domain response for subcarrier $i$ within block $k$, and $N$ the total number of subcarriers in the system.

In addition, we consider that the SNR gap per block is superior or equal to 1 , and consequently, to reach the target throughput, we should have

$$
E \geq \frac{3 N_{0}}{L} \sum_{i=1}^{L}\left(1 /\left|h_{k, i}\right|^{2}\right), \quad \forall k .
$$

\section{1) Case of a 2-subcarrier system}

In order to solve (6), we start with a simplified system with only $N=2$ subcarriers, and we try to minimize its mean BER. Two system configurations are possible, a 1-block system $(K=1, L=2)$, and a 2-block system $(K=2, L=1)$. The mean BER of the 1-block system is given from (6) by

$$
B E R_{1}=\frac{1}{2} \operatorname{erfc}\left(\sqrt{\frac{E N / 2 N_{0}}{1 /\left|h_{1}\right|^{2}+1 /\left|h_{2}\right|^{2}}}\right), E \geq \frac{3 N_{0}}{N}\left(\frac{1}{\left|h_{1}\right|^{2}}+\frac{1}{\left|h_{2}\right|^{2}}\right),(8)
$$

with $h_{1}$ and $h_{2}$ the frequency responses for the two subcarriers. The mean BER of the 2-block system is given by

$$
B E R_{2}=\frac{1}{4} \operatorname{erfc}\left(\sqrt{\frac{E N}{4 N_{0}}\left|h_{1}\right|^{2}}\right)+\frac{1}{4} \operatorname{erfc}\left(\sqrt{\frac{E N}{4 N_{0}}\left|h_{2}\right|^{2}}\right),
$$

$$
\text { with } E \geq \frac{6 N_{0}}{N\left|h_{1}\right|^{2}} \text { and } E \geq \frac{6 N_{0}}{N\left|h_{2}\right|^{2}} .
$$

Let $\left|h_{1}\right| \leq\left|h_{2}\right|$, then if the condition $E \geq 6 N_{0} / N\left|h_{1}\right|^{2}$ is true, all the conditions in (8) and (9) are true. Consequently, let $x$ and $y$ be two real numbers such that

$$
\begin{array}{lll}
E=6 N_{0} x / N\left|h_{1}\right|^{2} & \text { with } & x \geq 1, \\
y=\left|h_{1}\right|^{2} /\left|h_{2}\right|^{2} & \text { with } & 0<y \leq 1 .
\end{array}
$$

The difference $B E R_{1}-B E R_{2}$ can be expressed as

$$
f(y)=\frac{1}{2} \operatorname{erfc}\left(\sqrt{\frac{3 x}{1+y}}\right)-\frac{1}{4} \operatorname{erfc}\left(\sqrt{\frac{3 x}{2}}\right)-\frac{1}{4} \operatorname{erfc}\left(\sqrt{\frac{3 x}{2 y}}\right) .
$$

Function $f$ is strictly increasing, and for $y=1, f(y)=0$, consequently $f(y) \leq 0, \forall 0<y \leq 1$ and $B E R_{1} \leq B E R_{2}$.

Hence, in order to minimize the mean BER, the two subcarriers should be linked together with a spreading code, i.e. one single block should be used $(K=1, L=2)$.

2) Case of a multi-subcarrier system

Let us now consider two blocks $a$ and $b$ of length $L$ each. The objective is to know if the two blocks should be merged into one single block or kept separated, in order to minimize the mean BER. The BERs for a single-block system and a 2block system are given from (6) by

$$
\begin{gathered}
B E R_{K=1}=\frac{1}{2} \operatorname{erfc}\left(\sqrt{\frac{E N / 2 N_{0}}{\sum_{i=1}^{L}\left(1 /\left|h_{a, i}\right|^{2}\right)+\sum_{j=1}^{L}\left(1 /\left|h_{b, j}\right|^{2}\right)}}\right), \\
B E R_{K=2}=\frac{1}{4} \operatorname{erfc}\left(\sqrt{\frac{E N / 4 N_{0}}{\sum_{i=1}^{L}\left(1 /\left|h_{a, i}\right|^{2}\right)}}\right)+\frac{1}{4} \operatorname{erfc}\left(\sqrt{\frac{E N / 4 N_{0}}{\sum_{j=1}^{L}\left(1 /\left|h_{b, j}\right|^{2}\right)}}\right) \cdot(13)
\end{gathered}
$$

Let $H_{a}$ and $H_{b}$ be two real positive numbers such that

$$
1 / H_{a}^{2}=\sum_{i=1}^{L}\left(1 /\left|h_{a, i}\right|^{2}\right) \text { and } 1 / H_{b}^{2}=\sum_{j=1}^{L}\left(1 /\left|h_{b, j}\right|^{2}\right) \text {. (14) }
$$

If we put $H_{a}$ and $H_{b}$ in (12) and (13), these two equations become the same as (8) and (9), respectively, with $h_{1}=H_{a}$ and $h_{2}=H_{b}$. Then, the same results as with a 2-subcarrier system are obtained, and consequently $B E R_{K=1} \leq B E R_{K=2}$. Thus, grouping these two blocks into a single block of length $2 L$ minimizes the mean BER. Hence, merging different blocks, and consequently different subcarriers, into a single block reduces the mean BER.

We conclude that, in order to minimize the mean BER of a LP-OFDM system with a target throughput and a QPSK constellation, without taking into account the channel coding, the spreading code length should be maximized, i.e. a single block system should be considered. In addition, this analytical study shows the importance of the LP component in reducing the system mean BER, and consequently improving the system performance as we will see later on in the global system study. This improvement is due to the energy gathering capability of 
the LP-OFDM which can exploit, contrarily to OFDM, the residual energy conveyed by each subcarrier [7].

\section{ADDITION OF THE MIMO COMPONENT}

\section{A. MIMO scheme}

A MIMO component is added to the LP-OFDM system to improve the system robustness, and consequently to increase the system range. The well known Alamouti scheme with two transmit and two receive antennas is chosen, since it provides full spatial diversity gain, with no inter-symbol interference (ISI) and with low complexity receivers thanks to the orthogonality of its matrix. The multiuser access is also performed with TFC, but contrarily to WiMedia, the frequency hopping from a sub-band to another occurs at the end of each two consecutive OFDM symbols, since the Alamouti scheme is classically applied in space and time per subcarrier.

\section{B. New realistic MIMO-UWB channel model}

In general, a channel modeling approach with correlation matrices is simple since it requires only a power delay profile and two correlation matrices. But in the case of UWB with very large bandwidth, the channel model requires a time partitioning into very narrow taps. Each tap then contains only a very small number of channel paths. Consequently, Rayleigh model can not be directly applied to the UWB case and MIMO channel matrix can not be derived from a Gaussian matrix. Besides, UWB channel can be partitioned into sub-bands of bandwidth equal to the channel coherence bandwidth. Unfortunately, this approach is difficult to be implemented due to the inter-sub-bands correlations.

In this paper, we propose a new realistic channel model for MIMO-UWB applications. A geometric statistic approach, similar to the one adopted by the spatial channel model of 3GPP/3GPP2 [9], is considered. In this approach, a MIMO channel response can be obtained from a SISO impulse response, the angle of departure (AoD) and angle of arrival (AoA) statistics, and the antennas geometry at the transmitter and the receiver. In the proposed UWB model, the SISO impulse response is chosen from the channel model adopted by IEEE 802.15.3a [10], which is a modified version of SalehValenzuela model for indoor channels. Then, the spatial component is based on the IEEE 802.11n model [11], where AoD and AoA of the different clusters follow a uniform distribution. A typical channel response realization of the proposed MIMO model in the case of two transmit and two receive antennas, for an antennas spacing of $1 \mathrm{~cm}$ at the transmitter and at the receiver, is given in Fig. 1.

\section{GLOBAL SYSTEM PERFORMANCE}

\section{A. System parameters}

After having studied analytically the LP component and presented the proposed MIMO scheme, we move to a global system study taking into account the different functions of the transmission chain. The main parameters of the LP-OFDM
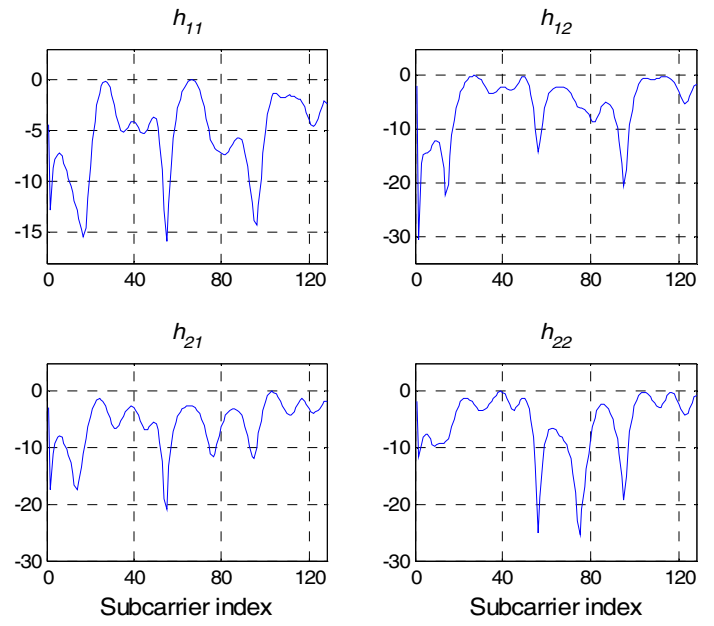

Fig. 1. Frequency response of a MIMO $2 \times 2$ channel over the first WiMedia sub-band, with an antennas spacing $d=1 \mathrm{~cm}$. Note that $h_{i j}$ represents the channel response between transmit antenna $i$ and receive antenna $j$.

system are listed in Table II.

Walsh-Hadamard orthogonal spreading codes and minimum mean square error (MMSE) single user detection are applied to limit the SI. Only Sylvester constructions of Hadamard matrices are chosen to simplify the LP-OFDM system. Compared to the WiMedia system, the number of data subcarriers is reduced from 100 to 96 for each OFDM symbol. Note that the remaining 4 subcarriers could be grouped into an additional block, to use 100 data subcarriers as in the WiMedia solution. In [12], it was shown that, for a LP-OFDM system with channel coding, the optimal value of $L$ that minimizes the system mean BER increases when the channel coding rate $r$ increases. This means that when $r$ tends to 1 , which is equivalent to the case without channel coding, $L$ tends to its maximum possible value, and consequently the analytical results obtained in Section III are verified. A unique code length $L=16$ is chosen here for all the simulations since it seems to be a good compromise for the used coding rates, according to previous studies. Thus, the LPOFDM system is composed of 6 blocks of 16 subcarriers each.

One of the advantages of the LP component is that it provides a wide range of data rates due to the high flexibility brought by the joint assignment of the number $C$ of used codes and the coding rates $r$. For low and medium data rates using a QPSK constellation, different $r$ and $C$ combinations are chosen in order to obtain data rate levels close to the ones proposed by WiMedia (See Table I and Table III for WiMedia and LPOFDM systems data rates, respectively). At these data rates, the objective is to increase the system robustness, and consequently the system range. In addition, a 16-QAM constellation is considered in order to improve the system throughput, and four very high data rates from $614 \mathrm{Mbit} / \mathrm{s}$ to around $1 \mathrm{Gbit} / \mathrm{s}$ are proposed, listed in Table III. In this case, one or two additional bits could be needed for the analog-to-digital conversion of the UWB receiver. Hence, the radio-frequency front-end complexity of the receiver is not increased significantly compared to the WiMedia solution. 
TABLE II

LP-OFDM SYSTEM PARAMETERS

\begin{tabular}{lc}
\hline \hline & \\
Parameter & Value \\
\hline Number of data subcarriers $(N)$ & 96 \\
IFFT/FFT size & 128 \\
Sampling frequency & $528 \mathrm{MHz}$ \\
Subcarrier frequency spacing & $4.125 \mathrm{MHz}$ \\
IFFT/FFT period & $242.42 \mathrm{~ns}$ \\
Zero padding duration & $70.08 \mathrm{~ns}$ \\
Total symbol length & $312.5 \mathrm{~ns}$ \\
Spreading code length $(L)$ & 16 \\
\hline \hline
\end{tabular}

\section{B. Simulation results}

In this section, the results of the global LP-OFDM system simulations performed on the first three WiMedia sub-bands (3.1-4.7 GHz) for the IEEE 802.15.3a CM1 channel model (line-of-sight scenario), with SISO and MIMO schemes, are presented. Note that the power gain provided by the use of 2 receive antennas is not taken into account in the presented results. If we want to take it into account, all the BER curves of the MIMO systems have to be shifted $3 \mathrm{~dB}$ to the left.

In Fig. 2, WiMedia performance at the highest (480 Mbit/s) and lowest $(53.3 \mathrm{Mbit} / \mathrm{s})$ possible data rates is presented. LPOFDM using QPSK with data rates close to WiMedia ones (medium data rate at $460 \mathrm{Mbit} / \mathrm{s}$, and low data rate at 51.2 $\mathrm{Mbit} / \mathrm{s}$ ) is also given with TFC for comparison. We can see that the LP component of a SISO system brings around $1.1 \mathrm{~dB}$ gain at medium data rate, and around $0.6 \mathrm{~dB}$ at low data rate for $B E R=10^{-4}$. In addition, MIMO LP-OFDM simulation results with the proposed realistic MIMO-UWB channel are presented for different spacing values $d$ between the two transmit antennas and between the two receive antennas. With an antennas spacing of only $1 \mathrm{~cm}\left(d \approx 0.13 \lambda_{c}\right.$, with $\lambda_{c}$ the center frequency wavelength), the MIMO scheme offers an additional $3.5 \mathrm{~dB}$ gain at medium data rate (for $B E R=10^{-4}$ ), resulting in a total gain of $4.6 \mathrm{~dB}$ compared to WiMedia. When antennas spacing is increased to $d=5 \mathrm{~cm}$ $\left(d \approx 0.66 \lambda_{c}\right)$, more channels diversity is obtained and the system performance tends to the performance of a MIMO LPOFDM system using four fully decorrelated SISO channels IEEE 802.15.3a. Hence, a total gain of $5.2 \mathrm{~dB}$ is obtained.

In Fig. 3, simulation results of MIMO LP-OFDM systems using 16-QAM constellation and offering very high data rates from $614 \mathrm{Mbit} / \mathrm{s}$ up to around $1 \mathrm{Gbit} / \mathrm{s}$, are presented. Note that the performance of the MIMO LP-OFDM system offering 1 $\mathrm{Gbit} / \mathrm{s}$ is even better than the performance of WiMedia which is providing only half of this data rate.

In Fig. 4, MIMO LP-OFDM results for all the proposed data rates are presented. The $E b / N_{0}$ required to obtain a $B E R=10^{-4}$ is given for each case and the gain offered by the use of TFC is evaluated. Here also, we can see the advantage of combining MIMO and LP-OFDM for UWB applications.
TABLE III

LP-OFDM SYSTEM DATA RATES

\begin{tabular}{cccc}
\hline \hline $\begin{array}{c}\text { Data rate } \\
(\mathrm{Mbit} / \mathrm{s})\end{array}$ & Modulation & $\begin{array}{c}\text { Convolutional } \\
\text { coding rate }(r)\end{array}$ & $\begin{array}{c}\text { Number of } \\
\text { codes }(C)\end{array}$ \\
\hline 51.2 & QPSK & $1 / 3$ & 4 \\
76.7 & QPSK & $1 / 3$ & 6 \\
115.1 & QPSK & $1 / 3$ & 9 \\
153.6 & QPSK & $1 / 3$ & 12 \\
192 & QPSK & $1 / 2$ & 10 \\
307 & QPSK & $1 / 2$ & 16 \\
409 & QPSK & $2 / 3$ & 16 \\
460 & QPSK & $3 / 4$ & 16 \\
614 & 16-QAM & $1 / 2$ & 16 \\
819 & 16-QAM & $2 / 3$ & 16 \\
921 & 16-QAM & $3 / 4$ & 16 \\
983 & 16-QAM & $4 / 5$ & \\
\hline \hline
\end{tabular}

At medium data rate (460 Mbit/s), a MIMO LP-OFDM system with $d=5 \mathrm{~cm}$ has a considerable gain of $5.2 \mathrm{~dB}$ compared to WiMedia as shown previously. In addition, a MIMO LP-OFDM system with $d=1 \mathrm{~cm}$ and without TFC has a gain of $2.7 \mathrm{~dB}$, and when applying TFC, an additional gain of 1.8 $\mathrm{dB}$ is obtained. However, we can notice that with $d=5 \mathrm{~cm}$, the TFC gain is only around $0.1 \mathrm{~dB}$. This is due to the fact that for $d=5 \mathrm{~cm}$, the MIMO and LP-OFDM components fully benefit from the total available diversity.

At high data rates we notice the importance of applying TFC whose gain increases significantly. In fact, at the highest data rate $(983 \mathrm{Mbit} / \mathrm{s})$, the TFC gain is equal to $3.9 \mathrm{~dB}$ with $d=1 \mathrm{~cm}$, and $1.3 \mathrm{~dB}$ with $d=5 \mathrm{~cm}$. In addition, at this data rate, MIMO LP-OFDM with $d=5 \mathrm{~cm}$ and with TFC is 0.6 $\mathrm{dB}$ better than WiMedia at $480 \mathrm{Mbit} / \mathrm{s}$.

\section{CONCLUSION}

In this paper, we have proposed a new MIMO LP-OFDM UWB system that can be seen as an extension of the well known WiMedia solution, and that does not significantly increase the system complexity compared to WiMedia. In addition, we have proposed a new realistic MIMO-UWB channel model. The analytical study led on the LP-OFDM waveform has confirmed the importance of the LP component in reducing the system mean BER, and consequently improving the system performance. The global system study has shown the advantage of combining MIMO and LP techniques in UWB, in terms of performance and flexibility.

For low and medium data rates, the proposed system outperforms WiMedia in terms of system robustness, and consequently in terms of system range. In addition, it can offer a data rate of around $1 \mathrm{Gbit} / \mathrm{s}$ with even better performance than WiMedia in terms of BER, which can provide at maximum only half of this data rate. In conclusion, the proposed MIMO LP-OFDM system can be advantageously exploited for UWB applications. 


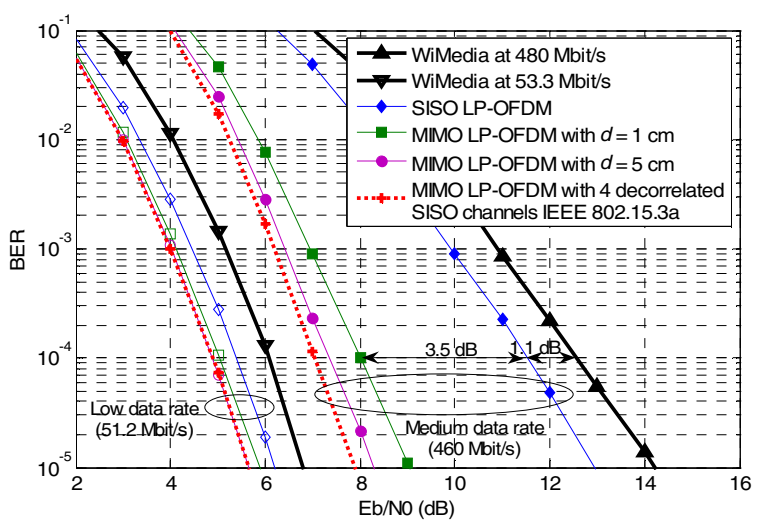

Fig. 2. Performance of SISO and MIMO LP-OFDM at low and medium data rates, with a QPSK constellation, for different antennas spacings $d$.

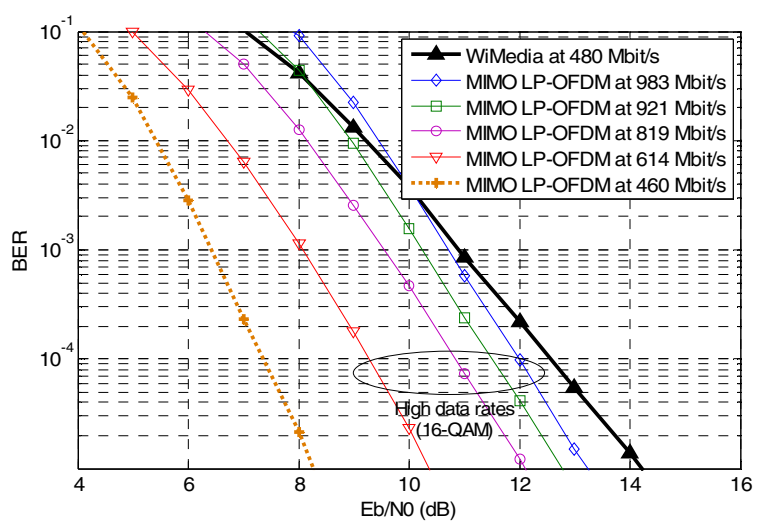

Fig. 3. Performance of MIMO LP-OFDM at high data rate with a 16-QAM constellation, for an antennas spacing of $5 \mathrm{~cm}$.

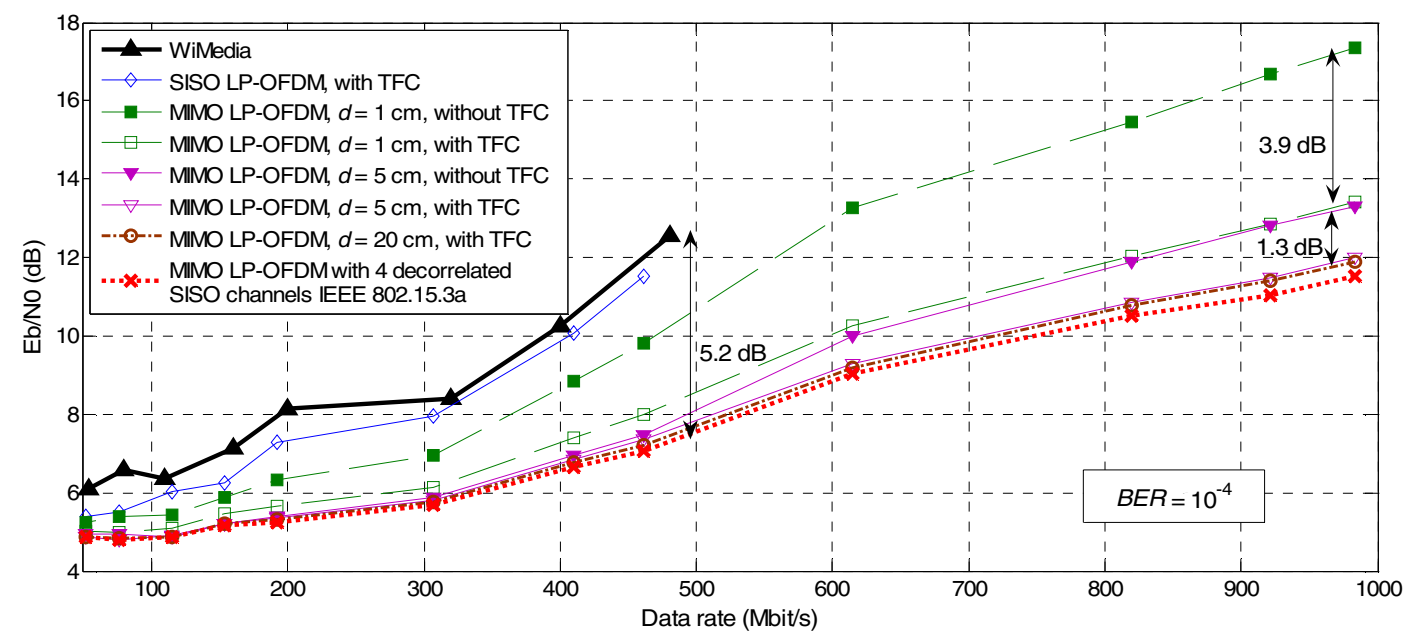

Fig. 4. Performance of SISO and MIMO LP-OFDM for the different proposed data rates, with and without TFC, at $B E R=10^{-4}$.

\section{ACKNOWLEDGMENT}

The authors would like to thank Dr. Louis-Marie Aubert for his work on the MIMO-UWB channel modeling.

\section{REFERENCES}

[1] "First report and order, revision of part 15 of the commission's rules regarding ultra-wideband transmission systems," FCC, ET Docket 98153, Feb. 14, 2002.

[2] A. Batra et al., "Multi-Band OFDM physical layer proposal for IEEE 802.15 task group 3a," IEEE document P802.15-04/0493r1, Texas Instruments et al., Sept. 2004.

[3] WiMedia Alliance Inc., "Multiband OFDM physical layer specification," Release 1.1, July 2005.

[4] Z. Wang and G. B. Giannakis, "Linearly precoded or coded OFDM against wireless channel fades?" in proc. IEEE 3rd Workshop on Signal Processing Advances in Wireless Commun. (SPAWC'01), pp. 267-270, China, March 2001.

[5] S. Kaiser and K. Fazel, "A flexible spread-spectrum multi-carrier multi- ple-access system for multi-media applications," in Proc. IEEE Intern. Symposium on Personal, Indoor and Mobile Radio Commun. (PIMRC'97), pp. 100-104, Finland, Sept. 1997.

[6] A. Stephan, J-Y. Baudais and J-F. Hélard, "Efficient allocation algorithms for multicarrier spread-spectrum schemes in UWB applications," in Proc. IEEE Intern. Conference on Ultra-Wideband (ICUWB'07), pp. 551-555, Singapore, Sept. 2007.

[7] M. Crussière, J-Y. Baudais and J-F. Hélard, "Adaptive spread-spectrum multicarrier multiple-access over wirelines," IEEE Journal on Selected Areas in Commun., vol. 24, pp. 1377-1388, July 2006.

[8] J. G. Proakis, Digital Communications, 4th Edition, McGraw-Hill, 2001.

[9] "Spatial channel model for multiple input multiple output (MIMO) simulations," 3GPP, TR 25.996 V6.1.0, Sept. 2003.

[10] J. Foerster, "Channel modeling sub-committee report (final)," IEEE P802.15.-02/490r1-SG3a, 2003.

[11] V. Ercerg et al., "TGn channel models," IEEE 802.11-03/940r4, May 2004.

[12] A. Stephan, E. Guéguen, M. Crussière, J-Y. Baudais and J-F. Hélard, "Optimization of linear precoded OFDM for high-data-rate UWB systems," EURASIP Journal on Wireless Commun. and Networking, vol. 2008, Article ID 317257, 2008. 